\title{
Monte Carlo simulations of X-ray absorption in the interstellar medium
}

\author{
Wiebke Eikmann ${ }^{* a b}$, Jörn Wilms ${ }^{a b}$, Randall K. Smith ${ }^{c}$, Julia C. Lee ${ }^{c}$ \\ ${ }^{a}$ Dr. Karl Remeis-Sternwarte Bamberg \\ Sternwartstrasse 7, 96049 Bamberg, Germany \\ ${ }^{b}$ Erlangen Centre for Astroparticle Physics (ECAP) \\ Erwin-Rommel-Str. 1, 91058 Erlangen, Germany \\ ${ }^{c}$ Center for Astrophysics (CfA), Harvard University, USA \\ Email: wiebke.eikmannesternwarte.uni-erlangen.de
}

\begin{abstract}
The shape and intensity of fluorescence lines is an important diagnostic tool to explore the characteristics of the material along the line of sight from an X-ray source. We present a Monte Carlo simulation of radiative transfer through a neutral gas, including photo absorption, fluorescent line emission, and Compton (down-)scattering. The primary spectrum is emitted by a source which is located either in the center of a spherical cloud or above a semi-infinite slab. In the former case, a pure transmitted spectrum is obtained while in the later case the reflected component is of interest. We present the dependence of intensity of the $\mathrm{Fe} \mathrm{K}_{\alpha}$ and $\mathrm{K}_{\beta}$ line and the centroid energy of the Compton shoulder as a function of $N_{\mathrm{H}}$. These calculations will be implemented in a revised version of the absorption model tbabs which includes lines emission and effective Compton down-scattering from the high energy range. This model is appropriate for column densities in excess of $10^{24} \mathrm{~cm}^{-2}$.
\end{abstract}

An INTEGRAL view of the high-energy sky (the first 10 years) - 9th INTEGRAL Workshop and celebration of the 10th anniversary of the launch

15-19 October 2012

Bibliotheque Nationale de France, Paris, France

\footnotetext{
* Speaker.
} 


\section{Computational methods}

Using Monte Carlo simulations we calculate how the passage through a potentially optically thick medium affects the shape of an incident continuum spectrum. The absorbing material is assumed to be neutral and uniformly distributed. The interaction mechanisms between photons and matter included in this model are photo absorption, fluorescent line emission, and Compton scattering. The photo absorption cross sections include all elements with $\mathrm{Z} \leq 30$. The code includes the emission of K- fluorescent lines according to fluorescent yields from Kaastra \& Mewe (1993) [1]. Multiple Compton scattering becomes important for $\tau_{\mathrm{T}} \geq 1$ (i.e., for $N_{\mathrm{H}} \geq 10^{24} \mathrm{~cm}^{-2}$ ). We include Compton scattering using the Klein Nishina formula and follow all photons (primary and fluorescent) until they leave the medium. In this way, Compton scattering is fully taken into account enabling us to model features like the Compton shoulders of the emission lines or the Compton bump at moderate energies. This work is an extension of earlier calculations by Matt [2].

\section{Transmission}

\subsection{General shape}

Figure 1 shows the absorption spectra of a powerlaw with photoindex $\gamma=2$ for different column densities. The fluorescent line emission is consistent in its self and is calculated simultaneously to the photo absorption. With increasing column density, the primary spectrum is more and more absorbed in the low energy range and the fluorescent lines becomes stronger. The flux in the high energy range is reduced by scattering out of the line of sight. The panel on the right shows the Fe-band, including the Compton shoulders of the iron and nickel lines.

\subsection{Geometry of the absorber}

The shape of the reprocessed spectrum strongly depends on the geometry of the absorbing material. Figure 2 shows the transmitted spectra for the same column density and two different absorbing geometries, a spherical geometry where the primary source is located at the center and a
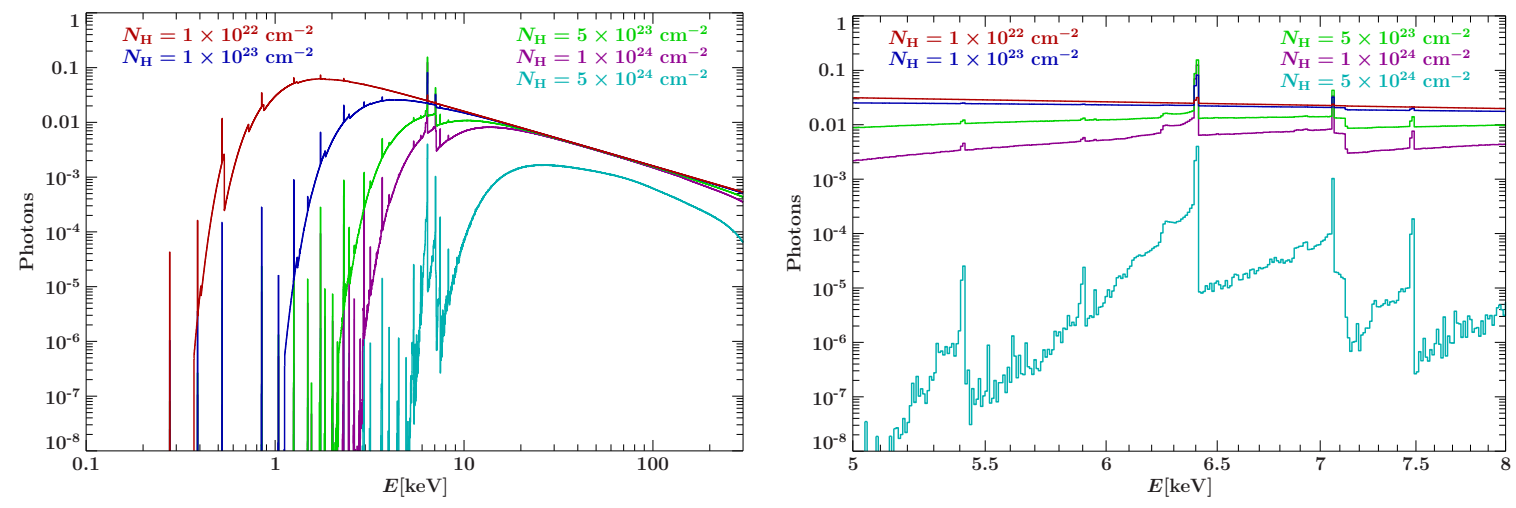

Figure 1: Left: Transmitted spectra calculated within a sphere geometry. With increasing $N_{\mathrm{H}}$, less photons escape in the low energy range and the various fluorescent lines become visible. The lack of photons in the high energy range is due to effective Compton down-scattering. Right: Zoom on the Fe-band. 


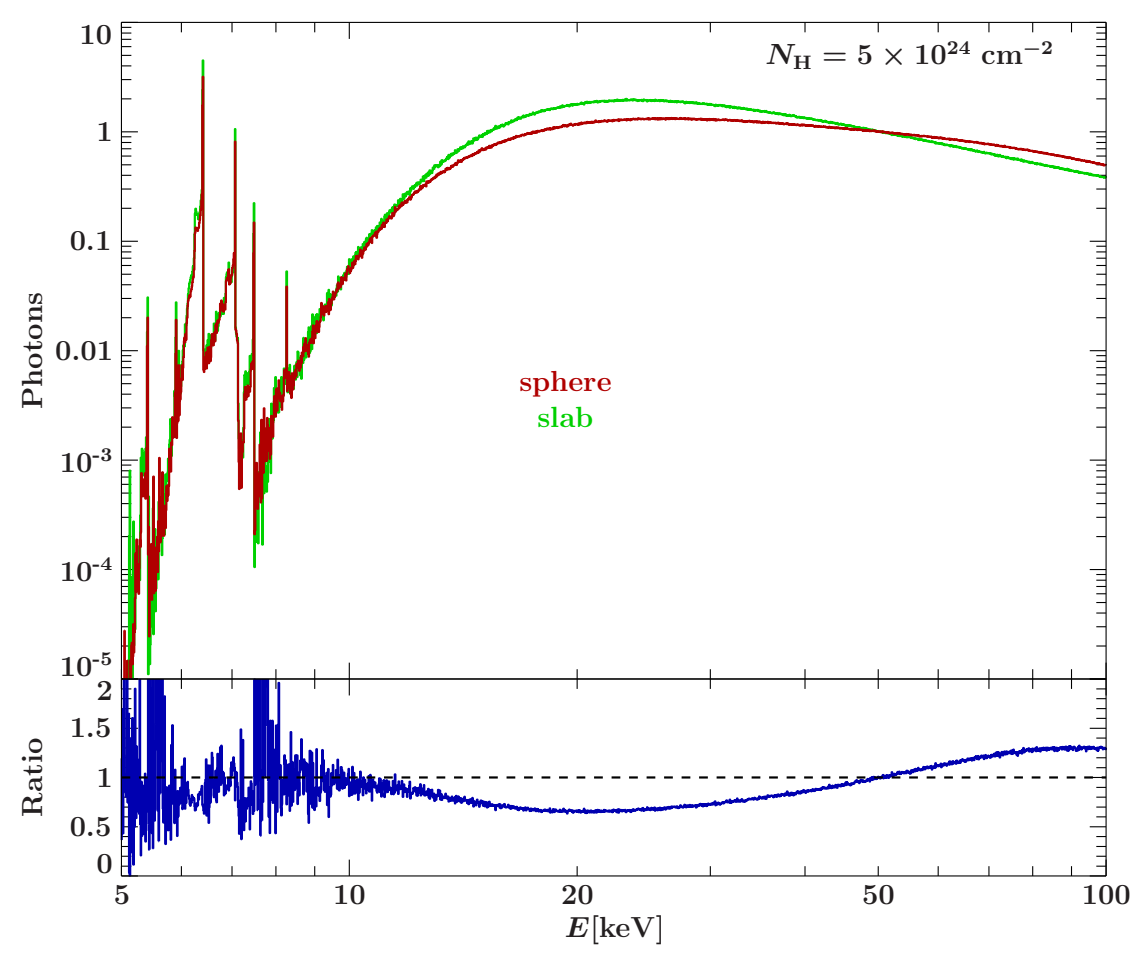

Figure 2: Absorbed spectra for transmission through a sphere (red) and though a slab (green) both calculated for $N_{\mathrm{H}}=5 \times 10^{24} \mathrm{~cm}^{-2}$

slab located between the observer and the source. The ratio between the spectra (lower panel) illustrates the geometry induced difference in the Compton shoulder and the Compton down scattering at higher energies.

\section{Reflection}

Figure 3 shows the reflected spectra for an input power law with photo index $\gamma=2.0$. In this picture, the photon source is located upon a semi-infinite slab and the spectra is averaged over all inclination angle. To demonstrate the importance of anisotropic Compton-scattering, the calculations were done once by using the Klein-Nishina formula (green curve) and once by considering only isotropic and elastic Thomson scattering (red curve). In the latter case, the photon energy remains unchanged during the scattering process. As Fig. 3 shows, the shape of the Compton hump between $10 \mathrm{keV}$ and $50 \mathrm{keV}$ is strongly affected by the choice of the scattering mechanism. 


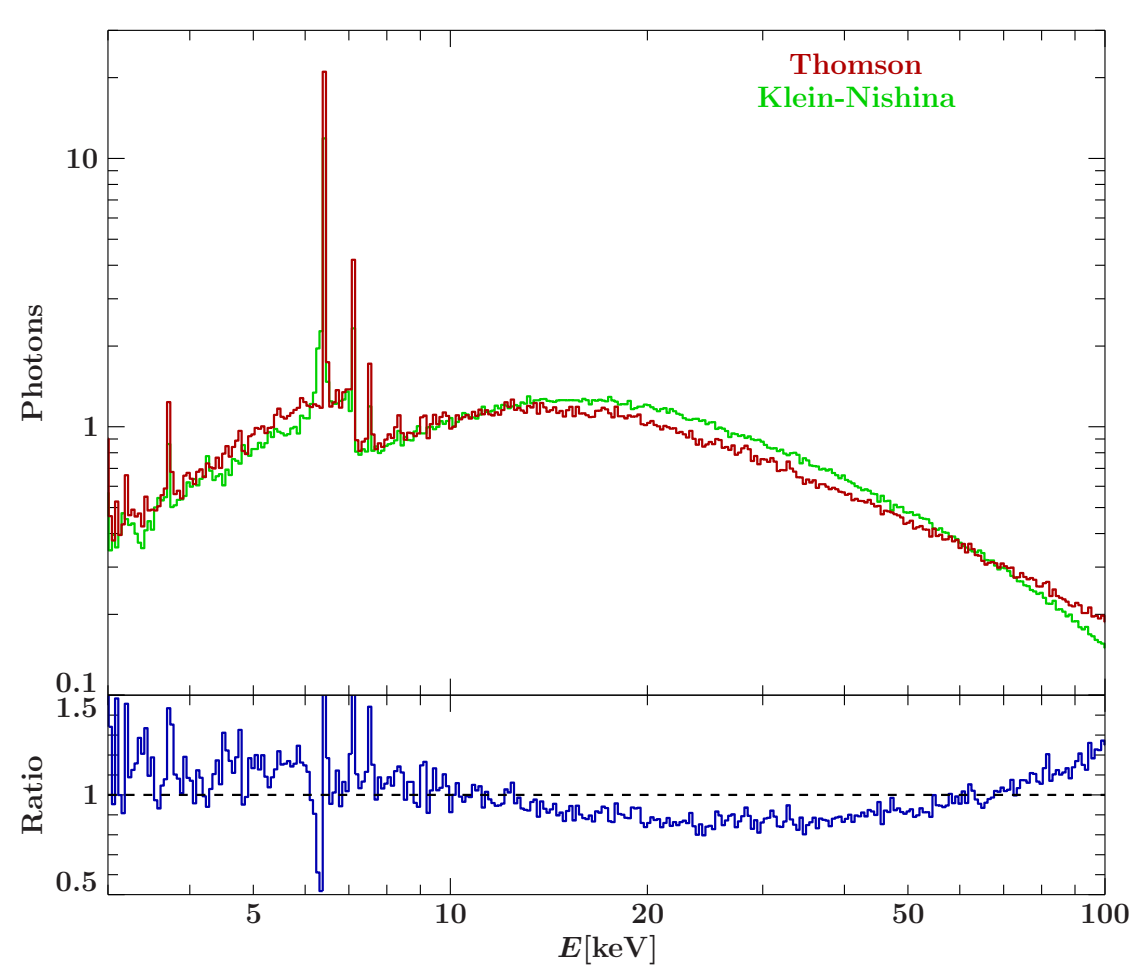

Figure 3: Reflected spectrum using the Thomson cross section (red) and the Klein-Nishina formula (green)
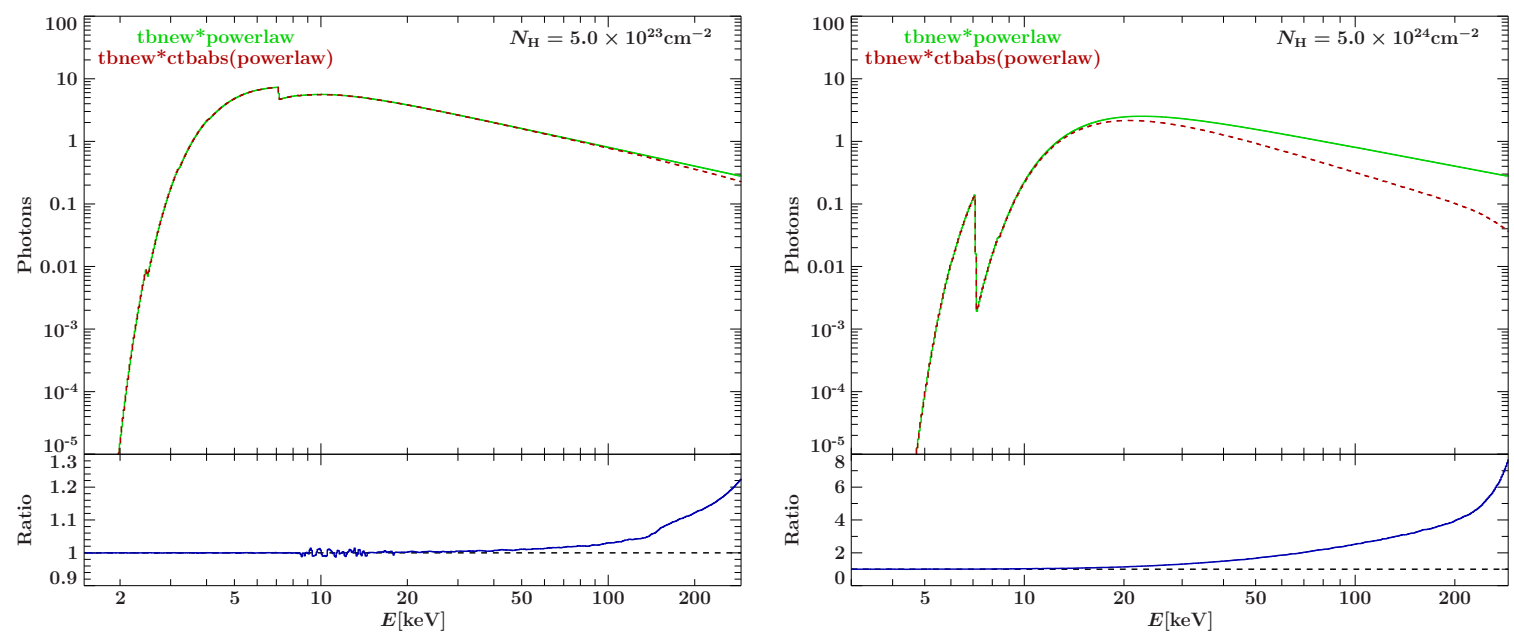

Figure 4: Comparison between the model tbabs with and without using a scattering model for different column densities. Note the strong effect of Compton scattering for large $N_{\mathrm{H}}$ 


\section{The effect of Compton scattering}

To demonstrate the influence of Compton scattering on the spectral shape we compared in Fig. 4 spectra that include Compton down scattering (red spectrum) with an absorbed power law model that does not include Compton scattering (green line). For moderate column densities (left panel) the models show the same behavior at low energies but there is a lack of photons at the high energy end of the spectrum. As the relative energy loss of a photon due to Compton scattering increases with increasing photon energy, the high energetic photons are effectively down scattered. For $N_{\mathrm{H}}$ larger than $10^{24} \mathrm{~cm}^{-2}$ (right plot) the residuals show a very significant deviation in this range.

\section{The shape of the iron K-lines}

Fluorescence lines offer an important diagnostic tool to determine the geometry, elemental abundances and other characteristics of the environment around compact objects. For the iron $\mathrm{K}_{\alpha}$ and $\mathrm{K}_{\beta}$ line, we calculate the intensity of the unscattered line photons (Fig. 5, top plot, abundances are those from Wilms et al., 2000 [4]). The line strength increases with $N_{\mathrm{H}}$ and has its maximum at $N_{\mathrm{H}} \sim 1 \times 10^{24} \mathrm{~cm}^{-2}$. Above this limit, more line photons are absorbed than emitted resulting in a decreasing line intensity. The lower panel shows the centroid energy of the complete Compton shoulder. The centroid energy remains nearly constant until the photons predominantly start to Compton scatter twice before leaving the medium.

\section{Outlook}

Both, Compton scattering and fluorescent line emission will be included in a future absorption model. In addition to the efforts described here, two further changes are currently being implemented into tbabs:

1. higher resolution cross sections close to relevant edges of $\mathrm{Ne}, \mathrm{O}$, and $\mathrm{Fe}$, making the model suitable for fitting gratings spectra.

2. the addition of mechanisms to include laboratory measured cross sections for solids expected to occur in dust in the ISM.

Test versions of these modifications will be available at

http://pulsar.sternwarte.uni-erlangen.de/wilms/research/tbabs 


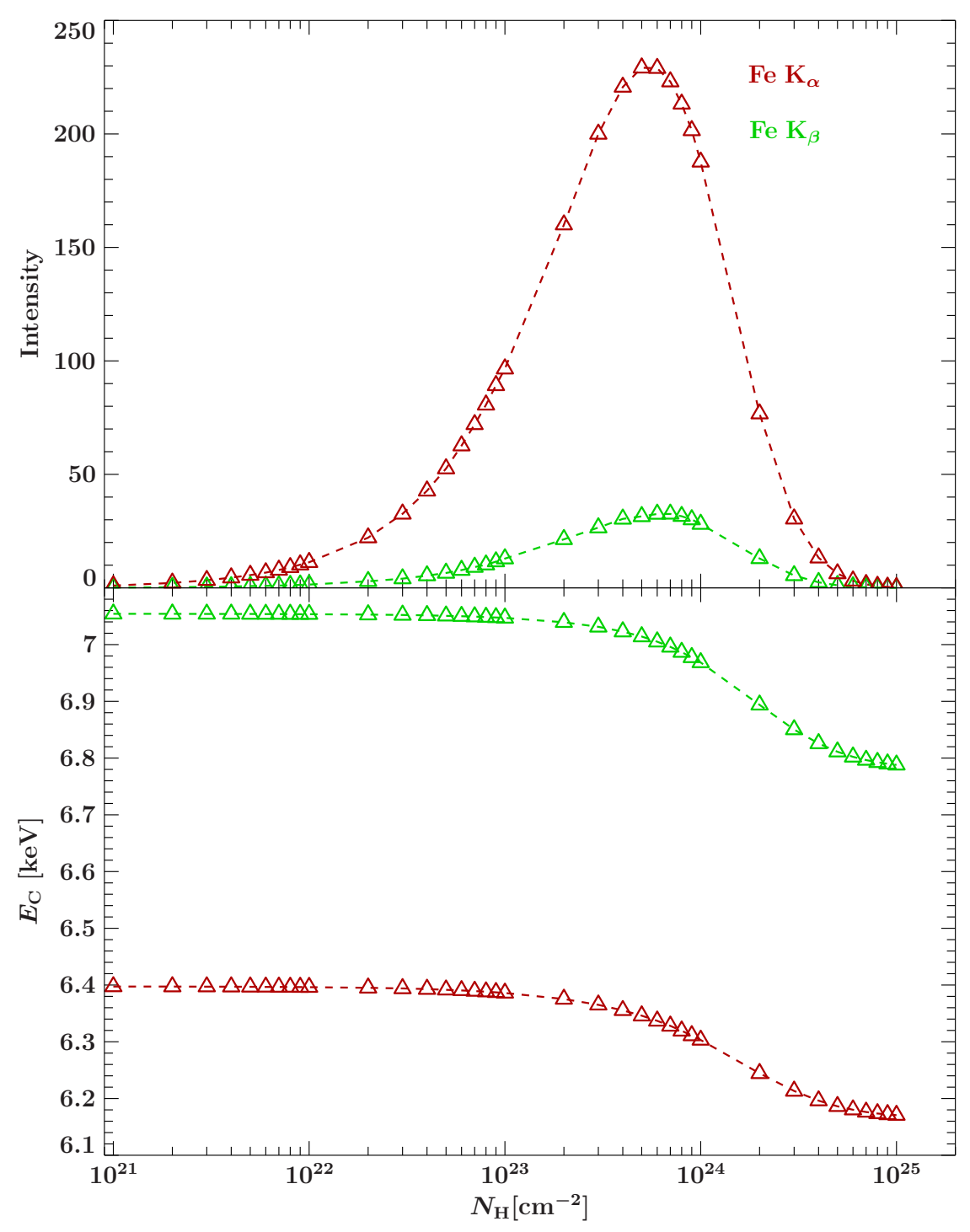

Figure 5: Line intensity and centroid energy $E_{\mathrm{C}}$ of the complete Compton shoulder of the iron lines

\section{References}

[1] Kaastra J. S., \& Mewe, R. 1993, Astron. Astrophys. Suppl. Ser., 97, 443

[2] Matt G. 2002, MNRAS, 337, 147

[3] Verner, D. A., \& Yakovlev, D. G. 1995, A\&A Suppl. 109, 125

[4] Wilms, J., Allen, A., \& McCray, R. 2000, ApJ, 542, 914 